\title{
Renta de agricultores y agroindustrias cooperados en Santa Catarina, Brasil
}

\section{Income of cooperated farmers and agroindustries in Santa Catarina, Brazil}

\author{
Rosaura Gazzola ${ }^{1 *}$; Geraldo da Silva e Souza ${ }^{2}$; Suellen Secchi Martinelli ${ }^{3}$ Anete Araújo de Sousa ${ }^{4}$; \\ Rossana Pacheco da Costa Proença ${ }^{5}$
}

${ }^{1}$ Doctora, Ingeniera Agrónoma, Empresa Brasileira de Pesquisa Agropecuária (Embrapa), Embrapa Sede, Brasilia; +55-61-3448-4283, rosaura.gazzola@embrapa.br; ${ }^{2}$ Doctor, Matemático y Economista, Empresa Brasileira de Pesquisa Agropecuária (Embrapa), Embrapa Sede, Brasilia; +55-613448-4194, geraldo.souza@embrapa.br; ${ }^{3}$ Doctora, Nutricionista y Matemática, Universidad Federal de Santa Catarina, Centro de Ciencias de la Salud, Núcleo de Pesquisa de Nutrição em Produção de Refeições, Departamento de Nutrición, Florianópolis; +55-48-3721-9784, suellen.martinelli@ufsc.br; ${ }^{4}$ Doctora Nutricionista, Universidad Federal de Santa Catarina, Centro de Ciencias de la Salud, Núcleo de Pesquisa de Nutrição em Produção de Refeições, Departamento de Nutrición, Florianópolis; +55-48-3721-9784, sousa.anete@gmail.com; ${ }^{5}$ Doctora, Nutricionista, Universidad Federal de Santa Catarina, Centro de Ciencias de la Salud, Núcleo de Pesquisa de Nutrição em Produção de Refeições , Departamento de Nutrición, Florianópolis; +55-48-3721-9784, rossana.costa@ufsc.br

\section{A R T I G O}

Recebido: $17 / 09 / 2018$

Aprovado: 28/11/2018
Palavras-chave:

Agricultura Orgánica

Alimentación Escolar

Políticas Públicas

PAA/PNAE

Supermercados.

Key words:

Organic Agriculture

School Feeding

Public Policies

PAA/PNAE

Supermarkets.

R E S U M O

El estudio ha analizado el impacto de programas públicos: Programa Nacional de Alimentación Escolar (PNAE) y Programa de Adquisición de Alimentos (PAA) en la renta bruta de agricultores y agroindustrias asociados en cooperativa, en Santa Catarina, sur de Brasil. Los datos estudiados son de 179 agricultores y 74 agroindustrias. Se modeló el ingreso medio de las ventas de los agricultores y agroindustrias, en función de la intensidad del uso de la agricultura orgánica, del tipo de venta (supermercados o ventas al gobierno) y tipo de alimento: alimentos procesados, frutas frescas, hortalizas in natura, productos de panadería y carne, huevos y lácteos. Para agricultores y agroindustrias es ventajoso vender alimentos procesados para supermercados. Para agricultores individuales es ventajoso vender frutas frescas y aumentar la proporción de ventas de productos orgánicos. Para las agroindustrias vender hortalizas resultaron en menor renta y la venta de productos orgánicos no afectan de manera significativa la renta de las agroindustrias.

\section{A B S T R A C T}

The study analyzed the impact of public programs: the National School Food Program (PNAE) and Food Acquisition Program (PAA) on the gross income of agribusinesses and family farmers marketing their products through cooperatives in Santa Catarina, southern Brazil. The data studied are 179 farmers and 74 agribusinesses. We model average sale returns per unit for farmers and agribusinesses as a function of the intensity of use of organic agriculture, type of sale: supermarkets or government programs, and type of food: fruits, vegetables, meat, eggs and dairy and bakery products. For both farmers and agribusinesses, we found to be more advantageous to sell processed foods to supermarkets. For farmers and agroindustries it is advantageous to sell processed foods for supermarkets. For individual farmers it is advantageous to sell fresh fruits and increase the proportion of sales of organic products. For agribusinesses, selling vegetables resulted in lower income and the sale of organic products does not significantly affect the income of the agroindustries.

\section{INTRODUCCIÓN}

En las zonas rurales la generación de ingresos está fundamentalmente vinculada a la producción agrícola, a los ingresos por trabajo fuera del establecimiento y a los ingresos generados en las actividades rurales no-agrícolas. Sin embargo, por lo general, está restringida por las dificultades que enfrentan los pequeños productores para vender en mercados más dinámicos (BUAINAIN, 2012).
La renta obtenida por aquellos puede tener su origen en el trabajo por cuenta propia o en el trabajo a sueldo, y éstas dos fuentes de ingreso pueden ser agrícolas o no-agrícolas. La renta se puede elevar, o por el aumento de la productividad, o por el aumento de la participación en el mercado de trabajo agrícola o por la subida del sueldo. La renta no agrícola es afectada por determinantes individuales y geográficos como acceso al empleo no agrícola. Las transferencias de renta son otra fuente de renta. Una familia puede recibir transferencias

\section{Revista Verde}

ISSN 1981-8203

Pombal, Paraíba, Brasil v. 14, n.1, jan.-mar, p.69-77, 2019 doi: $10.18378 /$ rvads.v14i1.5977 
privadas, como remesas o regalos, o transferencias púbicas, como la seguridad social o transferencias condicionales de renta en dinero (BUAINAIN, 2012).

Entre las dificultades que tienen los agricultores, se encuentran las deficiencias tecnológicas y la calidad de los productos (MALUF, 2004), razones por las que muchos agricultores no tienen acceso ni participan de los mercados, lo que es uno de los determinantes históricos de la pobreza rural en Brasil (MALUF et al., 2011).

Una alternativa para estrechar las relaciones entre la producción y el consumo es la creación de redes alternativas, llamadas cadenas cortas de producción y comercialización (MALUF, 2004; SCHRÖETTER et al., 2013), tales como marketing directo como ferias hortícolas; venta en mercados locales, donde la producción se dirige a los mercados institucionales (comida en las escuelas públicas, guarderías, hospitales y similares); así como, venta en mercados ampliados, con venta de productos certificados.

$\mathrm{Al}$ examinar la agricultura orgánica, realizada por pequeños agricultores de Brasil, Buainain (2012) sugiere que un buen ejemplo de la integración de estos agricultores en el mercado de consumo es el Programa de Adquisición de Alimentos (PAA), una política pública que articula el acceso al mercado, con incentivos para aumentar la producción y la productividad de los agricultores familiares.

Dicho programa (PAA) fue establecido hace más de 10 años por la Ley $\mathrm{n}^{\circ} 10.696 / 2003$ (BRASIL, 2003), pero el artículo 19 de esta Ley fue reglamentado en 2012 por el Decreto $n^{\text {o }} 7.775$ (BRASIL, 2012).

Durante estos años se han hecho varios cambios, surgieron nuevas definiciones y se han modificado los valores para las compras. También se han mantenido las modalidades de 'Compra con Donación Simultánea', que es la compra de los alimentos y donación a entidades de la red social de asistencia, y redes de enseñanza pública y filantrópicas. También se ha mantenido la 'Compra Directa', que tiene como objetivo apoyar los precios, satisfacer las demandas de los programas de acceso a la alimentación y de las redes de asistencia social, así como constituir y mantener almacenes públicos.

Sambuichi et al. (2014) en un estudio sobre el PAA y el PNAE, muestran que, la producción orgánica empezó a ser incentivada por el PAA a partir del año 2004, debido a la compra por un precio diferenciado. Ha sido permitido un límite de $30 \%$ de sobreprecio en el precio de los productos orgánicos y con adquisiciones informadas en separado de las convencionales. En el año 2013, ha sido estimada una meta para el PAA una compra de por lo menos 5\% de productos orgánicos para municipios y provincias en la modalidad "Donación Simultánea".

El PAA, por tener un techo inferior y ser menos exigente cuanto a forma y regularidad de suministro de productos, tiene más potencial para agricultores menos organizados y menos inseridos en el mercado, además de alcanzar también consumidores tales como instituciones educacionales menos formales, como guarderías y escuelas comunitarias donde el PNAE no llega (RIBEIRO et al., 2013).

Se suma al PAA, la Ley $n^{\circ} 11.947$ (BRASIL, 2009b) y la Resolución/CD/FNDE n 26/2013 (BRASIL, 2009a, 2013), que obliga a la compra de, al menos, el $30 \%$ de productos de la llamada agricultura familiar, preferentemente orgánicos, para el Programa Nacional de Alimentación Escolar (PNAE).
Dicho programa ofrece alimentación a los alumnos de escuelas públicas. La transferencia de este fondo se hace directamente a la escuela durante 10 meses del año escolar. En 2015, el presupuesto para el programa ha sido de $\mathrm{R} \$ 3,759$ mil millones en beneficio de 41,5 millones de estudiantes de educación básica (guarderías, escuela primaria, escuela secundaria y educación de adultos) (BRASIL, 2019).

Resáltese que la adquisición debe ser de productos alimenticios diversificados y de producción local, preferiblemente orgánicos, además de otras características. Ribeiro et al. (2013) afirman que el PNAE proporciona incentivo y desarrollo de la pequeña propiedad rural, creando mecanismos para fortalecer los agricultores familiares y el desarrollo local, además de la formación de hábitos saludables de alimentación.

En cuanto a las similitudes entre el PNAE y el PAA, Sambuichi et al. (2014), muestran su origen común y afirman que sus diferencias permiten que se completen, ya que miran a públicos diferentes de productores y consumidores.

La interfaz entre la agricultura familiar y el PNAE ha sido tema de estudio de diversos autores como Triches y Schneider (2010); Grisa (2010); Grisa et al. (2011a), Triches et al. (2019). Nuestro estudio alcanza en parte, lapso citado por Grisa et al. (2011b).

Estos autores, entre otros temas, hacen hincapié en la necesidad de profundizar el estudio del impacto económico del PNAE en unidades familiares que producen alimentos y en hogares que reciben alimentos. También Kroth et al. (2019) creen que es necesario investigar junto a los demás actores del PNAE, principalmente cooperativas y agricultores familiares, ya que en su estudio solo han podido abarcar un actor del PNAE: los ayuntamientos.

En este contexto, el objetivo de este estudio ha sido analizar los ingresos brutos de productores y de agroindustrias reunidos en cooperativa (municipios del estado de Santa Catarina, sur de Brasil), que venden alimentos orgánicos y convencionales, a supermercados y a programas del gobierno (PAA+PNAE) en dos diferentes años (2010 y 2011). El análisis ha sido realizado estudiando los factores que influyen en la renta de productores y agroindustrias.

\section{MATERIAL Y MÉTODOS}

La cooperativa analizada tiene socios en diversos municipios de Santa Catarina, entre ellos: Anitápolis, Gravatal, Rancho Queimado, Río Fortuna y Santa Rosa de Lima. Dicho territorio es denominado "Laderas de la Sierra General". Además de los socios en el territorio, todavía cuenta con socios en los municipios de Imaruí y Laguna. La provincia es Santa Catarina, sur de Brasil.

Los datos provienen de dos tipos de planillas suministrados por la cooperativa: alimentos entregados por agroindustrias y alimentos entregados por productores individuales. Estas planillas están separadas también en ventas a supermercados y ventas a los programas de gobierno (sumados los programas PAA+PNAE), detallados los orgánicos. Así como, hay planillas para el año de 2010 y 2011. Los datos que la cooperativa ha suministrado, corresponden a un total de 149 productores y 74 agroindustrias en 2010 y 2011.

Para el análisis de los datos de los agricultores individuales, los alimentos han sido clasificados, en primer lugar, en cinco grupos de alimentos: alimentos procesados 
(conservas, productos higienizados, mermeladas, azúcar, miel, pulpa de frutas, etc.); frutas frescas y hortalizas in natura.

Para el estudio de las agroindustrias hay dos factores más: carnes, huevos y lácteos y productos de panadería (pan, bizcocho, pasta fresca). El tipo de venta estudiado han sido las ventas a programas del gobierno (PAA+PNAE) o supermercados.

En las planillas constan los siguientes datos: alimento entregado, en seguida su valor bruto unitario, luego el valor neto unitario y al final de la planilla el valor bruto total. La renta bruta unitaria del agricultor $i$ o agroindustria $i$ es definida por la ecuación 1.

$$
R_{i}=\sum_{j=1}^{k} w_{j}^{i} \cdot p_{j}^{i}
$$

En que: $p_{1}^{i}, p_{2}^{i}, \ldots, p_{k}^{i}$ son los precios de los productos comercializados por el agricultor o agroindustria (cesta de productos de venta del productor o agroindustria $i$ ) y, $w_{j}^{i}$ es el peso del producto $j$ en esta cesta (ecuación 2).

$w_{j}^{i}=\frac{\text { renta del producto } j \text { para el productor o agroindustria } i}{\text { total de la renta del productor o agroindustria } i}$ (Ecu. 2)

Para analizar la venta de productos orgánicos frente a la venta de productos convencionales, se ha utilizado el ratio entre orgánicos y convencionales (ecuación 3).

ratio orgánicos $=\frac{\text { cantidad orgánicos }}{\text { cantidad orgánicos+cantidad convencionales }}($ Ecu. 3)

Los análisis estadísticos y la presentación de resultados están divididos en los dos grupos de estudio: productores y agroindustrias.

Para el análisis estadístico de los efectos de interés, se tomaron como respuesta la ordenación (ranks) de la variable renta y como variables independientes la ordenación (ranks) de la variable tasa de orgánicos y las variables binarias asociadas a los efectos. Dos formulaciones son consideradas: productores y agroindustria. Para los productores, el modelo estadístico considerado ha sido expresado en la ecuación 4.

$$
\begin{aligned}
& Y_{i j k l m}=\alpha+\beta R_{i j k l m}+\gamma_{i}+\delta_{j}+\theta_{k}++\tau_{l}++v_{m}+(\gamma \delta)_{i j}+(\gamma \theta)_{i k}+ \\
& (\gamma \tau)_{i l+}(\gamma v)_{i m+(\delta \theta)_{j k+}+(\delta \tau)_{l m+\varepsilon_{i j k l m}}}
\end{aligned}
$$

En que:

$$
\alpha, \beta, \gamma_{i}, \delta_{j}, \theta_{k}, \tau_{l}, v_{m}
$$

son parámetros asociados respectivamente a una media general, la tasa de orgánicos $\mathrm{R}$ y a los factores año, frutas, hortalizas, alimentos procesados y tipo de venta. Los componentes $(\gamma \delta)_{i j},(\gamma \theta)_{i k},(\gamma \tau)_{i l},(\gamma v)_{i m},(\delta \theta)_{j k},(\delta \tau)_{j l},(\delta v)_{j m},(\tau v)_{l m}$ son parámetros asociados a las interacciones de estos factores.

La variable respuesta (transformación de rank de la

renta) está representada por Y. La componente $\boldsymbol{\varepsilon}_{i j k l m}$ es una medida de error (residuo) con media cero y variancia constante. La transformación en ranks dona al método estadístico utilizado propiedades robustas cuanto a la heteroscedasticidad y no normalidad (CONOVER, 1999).
El modelo estadístico para la agroindustria sigue el mismo orden de ideas presentado arriba y en la ecuación 4, pero además, incluye dos efectos adicionales (productos de panadería; carne, huevos y lácteos).

El suministro de alimentos para las ventas al gobierno, ha venido de 79 familias en 2010 y 87 familias en 2011 .

La técnica utilizada es similar al análisis factorial con interacción con un modelo de análisis de variancia con estructura análoga al delineamiento completamente casualizado. La significancia de los efectos ha sido accedida en los tests F como consta en las Tablas 3 y 7 . Los contrastes han sido evaluados por medio del test de Student (Tablas 7 y 8). Todos los tests asumen la formulación general de la Ecuación 4 para agricultores individuales. Para la agroindustria se ha seguido el mismo orden de ideas presentado en la ecuación 4, pero además, se han incluido dos (2) efectos adicionales (productos de panadería; carne, huevos y lácteos). Cálculos ejecutados por intermedio del PROC GLM del SAS 9.2.

\section{RESULTADOS Y DISCUSIÓN}

Los alimentos han sido separados en cinco clases, que están representadas en las Tablas 1 y 2. En la primera columna, se presentan las tres clases de alimentos que se han analizado para agricultores individuales y al lado derecho están los alimentos que se han contabilizado en esta clase. Se ha hecho la clasificación de alimentos para los dos años analizados ya que en el año de 2010 hay más alimentos procesados que se venden a los supermercados (Tabla 1). En el año siguiente (2011), Tabla 2, se ha mantenido la diversidad de alimentos en la clase alimentos procesados y ha aumentado la variedad de hortalizas in natura.

Los autores Ribeiro et al. (2013) y Nunes et al. (2018), consolidan cuatro clases de productos: hortalizas, frutas, productos de panadería y carnes, huevos y lácteos. En nuestro estudio, consolidamos una clase a más: alimentos procesados. Esta clase de procesados abarca las conservas, las mermeladas, las jaleas, pulpa de frutas, salsa de tomate; o sea, productos agrícolas transformados, con valor añadido (Tablas 1 y 2 ).

Tabla 1. Alimentos vendidos por agricultores individuales de Santa Catarina en el año 2010, separados en clases para análisis de la renta bruta.

\begin{tabular}{ll}
\multicolumn{1}{c}{ Clase } & Alimentos \\
\hline Frutas & Plátano, caqui, naranja, limón, sandía y fresas \\
frescas &
\end{tabular}

\begin{tabular}{ll}
\hline $\begin{array}{l}\text { Hortalizas } \\
\text { in natura }\end{array}$ & $\begin{array}{l}\text { Calabaza, mandioca, lechuga, boniato, } \\
\text { remolacha, cebolla, zanahoria, chayote } \\
\text { (Sechium edule), judías, maíz verde, pepinillo, } \\
\text { pimientos verdes, repollo }\end{array}$ \\
\hline & $\begin{array}{l}\text { Azúcar moreno, miel, melaza } \\
\text { Conservas de remolacha y de pepinillos, }\end{array}$ \\
Alimentos & $\begin{array}{l}\text { Dulce de frutas, dulce de leche } \\
\text { procesados }\end{array}$ \\
& $\begin{array}{l}\text { Harina de maíz, derivados de maíz } \\
\text { Pulpa de palmera (Butia capitata) }\end{array}$ \\
& Salsa de tomate \\
Zumo de limón
\end{tabular}


Tabla 2. Alimentos vendidos por agricultores individuales de Santa Catarina en el año 2011, agrupados en clases para análisis de la renta bruta.

\begin{tabular}{ll}
\hline \multicolumn{1}{c}{ Clase } & \multicolumn{1}{c}{ Alimentos } \\
\hline $\begin{array}{l}\text { Frutas } \\
\text { frescas }\end{array}$ & $\begin{array}{l}\text { Plátano, caqui, naranja, limón, fresas y } \\
\text { melocotón }\end{array}$ \\
\hline & Calabaza, mandioca, lechuga, boniato, \\
& patatas, remolacha, cebolla, \\
Hortalizas & zanahoria, chayote (Sechium edule), col, \\
in natura & judías, maíz verde, pepinillo, pimientos, \\
& repollo, salada higienizada, sopa de \\
& legumbres, perejil, tomates, judías verdes \\
\hline & Azúcar moreno, miel, melaza \\
& Conserva de remolacha, de legumbres y de \\
& pepinillo \\
Alimentos & Dulce de frutas, \\
procesados & Harina de maíz, derivados de maíz \\
& Pulpa de palmera (Butia capitata) \\
& Salsa de tomate \\
& Zumo concentrado
\end{tabular}

Las tablas de alimentos aquí consolidadas, comparadas con las listas de productos agrícolas suministrados para el PNAE en municipios del Nordeste (NUNES et al., 2018) y de 3 municipios de la provincia del Rio Grande del Sur (RIBEIRO et al., 2013), se observa que gran parte de los productos agrícolas coinciden con los productos comercializados por los agricultores cooperados y por las agroindustrias de nuestro estudio.

Cuando analizada la renta bruta de productores individuales, se ha encontrado una diferencia estadística altamente significativa en esta variable $(\mathrm{F}=6,82$ y $p$ valor $<0,0001)$.

Para mejor explicar la dinámica de renta, venta de productos directos por el agricultor $\mathrm{o}$ asociados en cooperativas, merece mención cuando se estudia la localidad o el territorio. Según Arfini (2006) citando Briand (2000), los productos típicos sirven como palanca del desarrollo local. Una de las principales características de los productos típicos es su fuerte vinculación con el territorio y, en particular, con la localidad que lo representa, a la que a menudo deben su nombre. Los investigadores franceses sintetizan en el término terroir (terruño) los conceptos de savoir (saber) y savoir faire (saber hacer), expresando bien la interacción que existe entre las características ambientales de un determinado territorio y el factor humano capaz de transferirlas al bien "producto típico". Este último debe entenderse, por tanto, como un bien resultado de la síntesis de diversos factores, en primer lugar la territorialidad y, a través del mecanismo de la reputación, la revalorización de la tradición y la cultura histórica tanto del territorio como de sus habitantes (ARFINI, 2006).

Teniendo en cuenta las redes de venta directa entre productor y consumidor, Buainain (2012) y Sage y Goldberg (2012), señalan que la geografía juega un papel importante en la región y las posibilidades de producción y formación de mercados viables para el productor. Además, Sambuichi et al. (2014) creen que la proximidad de productores y consumidores y el fortalecimiento de los mercados locales conllevan al desarrollo local y evitan la circulación de mercancías a grandes distancias.

Con el PNAE, Nunes et al. (2018), muestran en su estudio que ha sido despertada más valoración para la diversificación de la agricultura familiar, así como para la necesidad de organización colectiva, especialmente en cooperativas. Como muestra nuestro estudio, así como, Baccarin et al. (2017), la comercialización vía cooperativas, genera renta.

En las planillas de datos, se ha hecho el conteo de productos orgánicos y convencionales vendidos en los dos años y a los distintos públicos (gobierno y supermercados). Luego se ha calculado la tasa de alimentos orgánicos.

La estimativa del parámetro $\beta$ (tasa de orgánicos) para agricultores individuales, ha resultado en un valor t de 3,80 y un $p$ valor de 0,0002, altamente significativo.

Cuando se trata de la venta de productos orgánicos, esta es ventajosa cuando la venta es hecha por el agricultor individual: su renta aumenta significativamente, ya que existe una asociación positiva entre la tasa de orgánicos y la renta.

Sambuichi et al. (2014), creen que el sobreprecio del orgánico en las compras del gobierno es una importante estrategia para apoyar la transición para la agricultura orgánica. En nuestro estudio vemos que es importante para acrecer la renta.

Autores como Dimitri y Oberholtzer (2009), verificando la venta de orgánicos en el mercado estadounidense o Saquet et al. (2010) analizando la venta de orgánicos en el mercado de Paraná (BR); encontraron, a menudo, que la demanda de productos orgánicos era superior a la oferta. De hecho, en nuestro análisis hemos encontrado que los productores individuales alcanzan renta más alta con la venta de orgánicos, por lo visto, en función del comportamiento del mercado, que a más demanda, más altos precios. Asunto este largamente puntualizado en varios análisis donde los autores afirman que cuando se añade valor al producto, pues resulta en más renta para el productor (MALUF, 2004; Triches y Schneider, 2010).

Analizada la influencia de cada efecto en la renta bruta del productor, se ha encontrado que los factores que influyen en la renta de éstos, de manera significativa, son: el ratio orgánicos/convencionales, el tipo de venta (si al gobierno o a los supermercados), si vende o no alimentos procesados, así como, ha habido influencia en la renta: la venta de frutas en interacción con el año de venta (Tabla 3 ).

En la Tabla 3 se presenta la significancia estadística de los demás factores y de sus interacciones de segundo orden. Se infiere que: el tipo de venta y los alimentos procesados son significativos estadísticamente.

La comparación de la renta bruta unitaria de productores que venden alimentos procesados y productores que no venden muestra que hay diferencias estadísticas en favor de aquellos que venden alimentos procesados. La interacción significativa es año*frutas.

Triches et al. (2019) muestran que en locales donde las relaciones sociales son más próximas y los actores tienen más compromiso, las ventas al gobierno tienen más éxito. También concluyen que el cambio legal no es suficiente para ocurran las aproximaciones necesarias entre las entidades ejecutoras de las políticas públicas. Pero en el Nordeste, Nunes et al. (2018) encontraron que el PNAE ha contribuido para diversificación de la agricultura y para el aparecimiento de organizaciones como cooperativas y para la construcción de mercados locales y regionales. 
Tabla 3. Significancia estadística de las variables que afectan la renta bruta de productores.

\begin{tabular}{lcccc}
\hline \multicolumn{1}{c}{ Variable } & GL & Cuadrado medio & Valor F & Valor $p$ \\
\hline Año & 1 & $1.202,5571$ & 0,98 & $0,3246 \mathrm{~ns}$ \\
Tipo de venta & 1 & $12.461,6875$ & 10,13 & $0,0018 * *$ \\
Alimentos procesados & 1 & $24.948,8180$ & 20,28 & $<0,0001 * *$ \\
Frutas & 1 & 971,7748 & 0,79 & $0,3758 \mathrm{~ns}$ \\
Hortalizas & 1 & 404,9670 & 0,33 & $0,5671 \mathrm{~ns}$ \\
Año*tipo de venta & 1 & $1.033,0311$ & 0,84 & $0,3612 \mathrm{~ns}$ \\
Año*alimentos procesados & 1 & $1.422,8398$ & 1,16 & $0,2841 \mathrm{~ns}$ \\
Año*frutas & 1 & $5.512,0017$ & 4,48 & $0,0361 *$ \\
Año*hortalizas & 1 & 871,8061 & 0,71 & $0,4014 \mathrm{~ns}$ \\
Tipo de venta*alimentos procesados & 1 & 770,6215 & 0,63 & $0,4301 \mathrm{~ns}$ \\
Tipo de venta*frutas & 1 & 164,0300 & 0,13 & $0,7156 \mathrm{~ns}$ \\
Tipo de venta*hortalizas & 1 & $1.073,5195$ & 0,87 & $0,3519 \mathrm{~ns}$ \\
\hline
\end{tabular}

** Altamente significativo, * significativo, ns: no-significativo

Ya para Sambuichi et al. (2014), el PNAE ha ayudado a crear una nueva mentalidad sobre la alimentación escolar y ha contribuido para la aproximación entre la comunidad, nutricionistas, productores familiares e instituciones de asistencia técnica. También señalan que otro efecto importante observado en estudios realizados sobre dicho programa es el fortalecimiento de la organización de productores, que consideran una estrategia esencial para viabilizar la inserción de la pequeña y diversificada producción de estos agricultores en el mercado, ofreciendo a éstos más condiciones de competitividad y de estructura para añadir valor a los productos.

Efectivamente vemos en nuestro estudio que eso ha ocurrido en este caso, ya que la cooperativa hace el papel de mediador con el mercado y los productores producen alimentos con valor añadido (orgánicos, procesados y orgánicos procesados) insiriéndose en el mercado a parte del institucional solamente.

En la Tabla 4 se presentan los contrastes de la interacción significativa presentada en la Tabla 3. En ella se presenta el contraste entre las medias ajustadas (renta bruta) del productor que ha vendido y que no ha vendido frutas en los años 2010 y 2011.

Hay diferencias estadísticas significativas para los contrastes (2-4) y (3-4).

El contraste 2-4, compara los años 2010 y 2011 para productores que venden frutas. El resultado significativo y positivo, muestra ventaja para el parámetro 2 (año 2010 y venta de frutas). O sea, que la renta bruta del productor que ha vendido frutas en el año 2010 es superior al que ha vendido frutas en el 2011 (parámetro 4).

El contraste 3-4, compara (mismo año 2011) agricultores que han vendido y que no han vendido frutas. El resultado significativo y la señal negativa, muestra ventaja para el parámetro 4 (año 2011, venta frutas), o sea, que ha tenido la renta bruta aumentada el agricultor que ha vendido frutas en el año 2011.

Tabla 4. Análisis estadística de la interacción año*frutas. Los efectos son 1: año 2010-no vende frutas, 2: año 2010-vende frutas, 3: año 2011-no vende frutas, 4: año 2011-vende frutas.

\begin{tabular}{rcc}
\hline Contraste & Estadística test & Valor $p$ \\
\hline $1-2$ & $-0,5591$ & $0,5770 \mathrm{~ns}$ \\
$1-3$ & $-0,4182$ & $0,6757 \mathrm{~ns}$ \\
$1-4$ & 1,4627 & $0,1459 \mathrm{~ns}$ \\
$2-3$ & 0,1271 & $0,8990 \mathrm{~ns}$ \\
$2-4$ & 2,0296 & $0,0444^{*}$ \\
$3-4$ & $-2,2225$ & $0,0279^{*}$ \\
\hline
\end{tabular}

* Significativo, ns: no-significativo
El análisis de la renta bruta de agricultores individuales muestra que la venta de frutas aumenta la renta en los dos años analizados. Se observa que hay baja variedad de oferta de frutas (hay plátano, caqui, naranja, limón, sandía, fresas y melocotón en los dos años). De acuerdo con Ribeiro et al. (2013), estos autores han encontrado baja participación de los productores rurales en las ventas al PNAE, en la clase frutas y hortalizas, seguramente debido a la legislación y la dificultad en mantener una escala de producción de frutas y hortalizas en pequeñas propiedades rurales.

En cuanto al tipo de género alimenticio, Baccarin et al. (2017) (estudio de dados de Santa Catarina, Paraná y São Paulo), en líneas generales, han observado que predominaban productos de origen vegetal e in natura o mínimamente procesados, ya que estos tienen normas sanitarias más sencillas y no necesitan de equipos sofisticados para su procesamiento, al contrario de lo que se observa en productos de origen animal.

En nuestro estudio, hemos visto la renta bruta aumentar con la venta de frutas, fenómeno relacionado a la posibilidad que ofrece la cooperativa: juntar productos de agricultores individuales y hacerse un hueco en el mercado. Observe también la baja variedad de frutas vendidas frente a la variedad de hortalizas y alimentos procesados.

También han sido analizados los datos de las agroindustrias, que serán enseñados a partir de esta parte del texto. Las listas de ventas de las agroindustrias apuntan para más variedad de hortalizas en el año 2010 y más variedad de carnes en al año 2011. El hecho de que haya ocurrido este cambio, hemos encontrado explicación en el trabajo de Baccarin et al. (2017). Dicen los autores que han percibido que en Santa Catarina y Paraná, había más demanda por los ayuntamientos por productos de medio y alto grado de procesamiento, así como por productos de origen animal, cuando comparados con datos de São Paulo. Ellos creen que esto es fruto del nivel organizacional y de inversiones hechas en equipos industriales por parte de asociaciones y cooperados de los estados del sur.

Cuando comparadas las Tablas 5 (año 2010) y 6 (año 2011), observamos que las ventas del segmento carnes, huevos y lácteos, así como de procesados; tienen más variedad de alimentos en el año 2011 para las ventas al gobierno (en términos económicos también hay más renta) y que en el año 2010 hay más diversidad de oferta en el segmento de hortalizas in natura (Tabla 5). 
Tabla 5. Alimentos comercializados por agroindustrias de Santa Catarina en el año 2010, separados en clases para análisis de la renta bruta.

\begin{tabular}{ll}
\hline Clase & \multicolumn{1}{c}{ Alimentos } \\
\hline Carnes, huevos, lácteos & Filete de pescado, pollo rústico, leche, huevos \\
\hline Frutas frescas & Plátano, guayaba, naranja, limón, melón, fresa y melocotón \\
\hline \multirow{3}{*}{ Hortalizas in natura } & $\begin{array}{l}\text { Calabaza, mandioca, lechuga, ajo, boniato, patatas, remolacha, brócolis, cebolla, cebolla en rama, } \\
\text { zanahoria, chayote (Sechium edule), col minera, judías, maíz verde, pepinillo, rábanos, repollo, } \\
\text { rúcula, perejil, legumbres (en trozos), tomate }\end{array}$ \\
\hline Productos de panadería & Bizcocho de maíz y pasta casera \\
& $\begin{array}{l}\text { Azúcar en bruto, azúcar moreno, miel, melaza, plátano en pasas, } \\
\text { Arroz, }\end{array}$ \\
& $\begin{array}{l}\text { Conservas de remolacha, de brócolis, de cebolla, de zanahoria, de pepinillos y de judías verdes } \\
\text { Alimentos procesados }\end{array}$ \\
& $\begin{array}{l}\text { Dulces: de plátano, de palmera (Butia capitata), de higo, de naranja con pimienta, de leche, de } \\
\text { fresas }\end{array}$ \\
& Harina de mandioca \\
& $\begin{array}{l}\text { Mermeladas de mora, de palmera (Butia capitata), de naranja con pimienta, de fresas, de fresas con } \\
\text { pimienta, de melocotón, de physalis, de mandarina y de uva }\end{array}$ \\
& Salsa de tomate, picles, vinagre \\
& Zumo de maracuyá, zumo de uva
\end{tabular}

Tabla 6. Alimentos comercializados por agroindustrias de Santa Catarina en el año 2011, separados en clases para análisis de la renta bruta.

\begin{tabular}{ll}
\hline Clase & Corazón de pollo, muslos y sobre muslos, \\
\hline \multirow{2}{*}{ Carnes, huevos, lácteos } & muslo del ala, hígado de pollo, filete de pechuga de pollo, \\
& Pollo rústico, pollo rústico en mitades y entero, \\
& Buche de pollo, huevos, pechuga de pollo, queso colonial \\
\hline Frutas frescas & Piña, plátano, caqui, kiwi, naranja, limón, fresa, melocotón y uva \\
\hline \multirow{2}{*}{ Hortalizas in natura } & Calabaza, mandioca, lechuga, ajo, boniato, patatas, \\
& remolacha, cebolla, zanahoria, chayote (Sechium edule), judías, pepinillo, repollo y tomate \\
\hline Productos de panadería & Galletas y pasta artesanal \\
\hline & Azúcar en bruto, azúcar moreno, miel, melaza, plátano en pasas \\
& Conservas de remolacha, de brócolis, de cebolla, de zanahoria, de mini-maíz, de pepinillo y de \\
& judías verdes \\
& Fresas, higos y melocotón en almíbar \\
& Dulce de plátano, de fresas \\
Cátchup, harina de mandioca & Mermelada de piña, de mora, de palmera (Butia capitata), de guayaba, de naranja con pimienta, \\
de fresas, de fresas con pimienta, de melocotón, de physalis, de mandarinas y de uva. & Salsa de tomate, salsa de tomate con aceitunas, con champignon, con albahaca, sazonado con \\
pimienta y jengibre \\
Pulpa de palmera (Butia capitata), pulpa de tomate
\end{tabular}

En los trabajos de Ribeiro et al. (2013) y Nunes et al. (2018), así como en nuestro estudio aparecen siempre, melaza, miel; hortalizas y frutas y se nota también una relación directa entre cultura alimentar y productos ofertados al PNAE. De hecho, los agricultores y agroindustrias de la cooperativa analizada producen varias hortalizas en conserva, de acuerdo con su cultura y tradición.

Las ofertas al PNAE (y a los supermercados, en nuestro estudio) están relacionadas a los cultivos locales y a la tradición alimentar local.

Además de la producción de orgánicos, la diferencia que hemos encontrado en nuestro estudio ha sido la gran variedad de productos procesados por estos agricultores y, principalmente, por las agroindustrias estudiadas. Todos productos que llevan a acrecimos en la renta.

Tienen gran variedad de productos higienizados y productos en conserva, así como fabrican y comercializan productos diferenciados como la mermelada de naranja con pimienta, la salsa de tomates con hierbas o con pimienta o con jengibre. Autores como Schröetter et al. (2013), en su análisis de las cooperativas, creen que hay espacio para el crecimiento de la producción, sobre todo, en la especialización y en la estructuración para el suministro en los periodos entre cosechas. Este el caso de la cooperativa analizada.

En el análisis de la renta de las agroindustrias fueron considerados 5 clases de alimentos, dos tipos de ventas (al gobierno o a supermercados) en dos años distintos (2010 y 2011).

La renta de la agroindustria está significativamente influenciada por las variables analizadas. En el análisis de variancia se ha encontrado una diferencia estadística altamente significativa (Valor $\mathrm{F}=4,77$ y un $p$ valor de $<0,0001)$.

A partir de este resultado se ha estudiado la influencia de la venta de productos orgánicos en la renta de las agroindustrias. 
La estimativa del coeficiente lineal para la tasa de orgánicos, hemos encontrado que, para la renta de las agroindustrias no hay diferencias estadísticas significativas en el caso de que vendan productos orgánicos o alimentos convencionales. El valor de $\mathrm{F}$ es 1,78 con un $p$ valor de 0,1872, no significativo.

Con relación a productos orgánicos: analizados los dados de las agroindustrias, encontramos que, vender orgánicos no cambia su rentabilidad. Pero sí aumenta la renta (años estudiados) con la venta de productos procesados a los supermercados.

El mismo fenómeno aquí encontrado había sido descrito por Schröetter et al. (2013), donde estos autores han encontrado que los agricultores organizados en asociaciones o cooperativas, ayudaron a mejorar los mercados locales como el de las compras institucionales, yendo más allá de estos y consolidándose como proveedores de los supermercados.

En Ijuí-RS (municipio con aproximadamente 75.000 habitantes), la creación de asociaciones y cooperativas fue la respuesta hallada para superar las dificultades. En dicho local hay tres agroindustrias de panadería y dos de embutidos, haciendo más estable y segura la venta al PNAE (RIBEIRO et al., 2013).

Cuando analizadas las variables que influyen en la renta bruta de las agroindustrias, han sido halladas diferencias significativas en la renta bruta de éstas para la venta de alimentos procesados, para hortalizas y para la interacción tipo de venta*alimentos procesados. Los demás parámetros cuando comparados no muestran diferencias estadísticas entre ellos (Tabla 7). Tampoco hay diferencia entre los dos años de venta, o si venden frutas, productos de panadería o carnes, huevos y lácteos.

El principal resultado de la Tabla 7 es la interacción significativa: tipo de venta (para el gobierno o para los supermercados) y la venta de alimentos procesados o no procesados.

Tabla 7. Análisis estadística de las variables que afectan la renta bruta de las agroindustrias.

\begin{tabular}{lcccc}
\hline \multicolumn{1}{c}{ Variable } & GL & Cuadrado medio & Valor F & Valor $p$ \\
\hline Año & 1 & 207,6483 & 0,89 & $0,3498 \mathrm{~ns}$ \\
Tipo de venta & 1 & 224,3568 & 0,96 & $0,3313 \mathrm{~ns}$ \\
Alimentos procesados & 1 & $1.051,2143$ & 4,50 & $0,0384 *$ \\
Frutas & 1 & 30,2017 & 0,13 & $0,7205 \mathrm{~ns}$ \\
Hortalizas & 1 & $2.086,6312$ & 8,94 & $0,0042^{* *}$ \\
Panadería & 1 & 185,8152 & 0,80 & $0,3763 \mathrm{~ns}$ \\
Carnes, huevos y lácteos & 1 & 533,7411 & 2,29 & $0,1364 \mathrm{~ns}$ \\
Año*tipo de venta & 1 & 78,3532 & 0,34 & $0,5648 \mathrm{~ns}$ \\
Año*alimentos procesados & 1 & 672,8186 & 2,88 & $0,0953 \mathrm{~ns}$ \\
Año*frutas & 1 & 308,7964 & 1,32 & $0,2552 \mathrm{~ns}$ \\
Año*hortalizas & 1 & 81,4496 & 0,35 & $0,5572 \mathrm{~ns}$ \\
Año*panadería & 1 & 130,7319 & 0,56 & $0,4575 \mathrm{~ns}$ \\
Año*carnes, huevos, lácteos & 1 & 59,2200 & 0,25 & $0,6166 \mathrm{~ns}$ \\
Tipo de venta*alimentos procesados & 1 & $1.530,1783$ & 6,55 & $0,0133 *$ \\
Tipo de venta*frutas & 1 & 236,7576 & 1,01 & $0,3184 \mathrm{~ns}$ \\
Tipo de venta*hortalizas & 1 & 23,8152 & 0,10 & $0,7507 \mathrm{~ns}$ \\
Tipo de venta*panadería & 1 & 60,2783 & 0,26 & $0,6134 \mathrm{~ns}$ \\
Tipo venta*carnes, huevos, lácteos & 1 & 146,2267 & 0,63 & $0,4322 \mathrm{~ns}$ \\
\hline
\end{tabular}

** Altamente significativo, * significativo, ns: no-significativo

Curiosamente la venta de hortalizas disminuye la renta de las agroindustrias. Esto se explica porque el principal peso en la renta es la venta de productos procesados. Añadir valor a las hortalizas, venderlas en conserva, higienizadas, salsas, congeladas, etc., genera renta.

Autores como Maluf (2004), Triches y Schneider (2010), Ribeiro et al. (2013) y Kroht et al. (2019), encuentran en sus análisis que al añadir valor a los productos, el agricultor (o agroindustria) se diferencia en el mercado y penetra en nichos donde no entraria caso no hiciera de esta manera.

Lo que si cabe apuntar aquí es que al ofrecer productos al PNAE o al PAA, el agricultor ha hecho su parte especializándose y cooperativándose, y así de esta manera ha podido entrar no solo en el mercado institucional, pero tambien en los supermercados. Claro está que cuando transforma el producto original en conservas o mermeladas o frutas en almíbar o salsas, no solo ocupa un hueco en el mercado, pero también obtiene renta.
La interacción tipo de venta*alimentos procesados ha sido analizada para estudiar cual factor es el que más pesa en la renta de las agroindustrias.

El análisis estadístico que se presenta en la Tabla 8, muestra que la venta de alimentos procesados a los supermercados (parámetro 4) aumenta significativamente la renta de las agroindustrias. Hay diferencias estadísticas significativas en los contrastes: (1-4), (2-4) y (3-4).

La señal negativa del test muestra la ventaja del parámetro 4 en relación a 1, 2 y 3 .

El análisis apunta que el 4 (agroindustria vender a supermercados productos procesados) tiene más renta que el 1 (agroindustria vender al gobierno productos no procesados), que el 2 (agroindustria vender al gobierno productos procesados) y que el 3 (agroindustria vender al supermercado, productos no procesados).

Nuestros análisis apuntan para aumento de la renta con ventas a supermercados. Otros autores encontraron resultados similares a nuestro estudio, como los realizados en Minas Gerais por Costa et al. (2015), en São Paulo por Ferreira de 
Souza-Esquerdo y Bergamasco (2014), en Rio Grande del Sur por Ribeiro et al. (2013) y Santos et al. (2014).

Tabla 8. Análisis estadístico de la interacción del tipo de venta*alimentos procesados. Los efectos son: 1: gobiernoalimentos no procesados, 2: gobierno-alimentos procesados, 3: supermercados-alimentos no procesados, 4: supermercados-alimentos procesados.

\begin{tabular}{ccc}
\hline Contraste & Estadística test & Valor $p$ \\
\hline $1-2$ & 0,2006 & $0,8417 \mathrm{~ns}$ \\
$1-3$ & 0,8791 & $0,3832 \mathrm{~ns}$ \\
$1-4$ & $-2,1121$ & $0,0393^{*}$ \\
$2-3$ & 0,6087 & $0,5453 \mathrm{~ns}$ \\
$2-4$ & $-2,2019$ & $0,0320^{*}$ \\
$3-4$ & $-3,5016$ & $0,0009^{* *}$ \\
\hline **Altamente significativo, *significativo, ns: no-significativo
\end{tabular}

Este hecho ha sido diagnosticado por Sambuichi et al. (2014), donde afirman que el techo de 20 mil reales del PNAE y sus exigencias más elevadas, tiende a restringir los suministradores a cooperativas y asociaciones de agricultura familiar más organizada. Lo que ha ocurrido en nuestro estudio, la cooperativa analizada no solo atiende a la red pública, pero también a supermercados, que es de donde sacan más renta.

Kroth et al. (2019) encuentran en su estudio que hay falta de oferta de productos para alimentación escolar (debido a varias dificultades encontradas) y apuntan que una de las formas de incentivo seria ampliar el valor disponible por aluno pago por el FNDE.

Autores que han estudiado el mismo PNAE, como Ribeiro et al. (2013), Santos el al. (2014), Triches et al. (2019), constatan lo mismo que hemos encontrado: el hecho de la sencilla publicación de la Ley en el año 2009, no trajo estos productos para las cantinas escolares de escuelas públicas de los grandes centros, así como, no todos municipios compran estos alimentos.

\section{CONCLUSIONES}

Para productores individuales y agroindustrias, es ventajoso vender a los supermercados y que los alimentos sean procesados.

Para los productores, mejora la renta cuando venden orgánicos frente a la venta de convencionales. Para las agroindustrias este factor no influye en la renta.

Para los productores individuales: vender frutas aumenta la renta y para las agroindustrias vender hortalizas, disminuye la renta.

\section{REFERENCIAS}

ARFINI, F. Productos típicos y desarrollo rural: entre calidad y políticas de gobernanza. Estudios Agrosociales y Pesqueros, n. 210, p. 13-38, 2006.

BACCARIN, J. G; TRICHES, R. M; TEO, C. R. P. A; PEREIRA DA SILVA, D. B. Indicadores de Avaliação das Compras da Agricultura Familiar para Alimentação Escolar no Paraná, Santa Catarina e São Paulo. RESR, Piracicaba-SP, v. 55, n 01, p. 103-122, 2017. http://dx.doi.org/10.1590/123456781806-94790550106.
BRASIL. Presidência da República. Casa Civil. Subchefia para Assuntos Jurídicos. Lei $n^{\circ}$. 10.696, de 2 de julho de 2003. Dispõe sobre a repactuação e o alongamento de dívidas oriundas de operações de crédito rural e dá outras providências. Diário Oficial da União; Poder Executivo, 2003.

BRASIL. Ministério da Educação. Fundo Nacional de Desenvolvimento da Educação. Conselho Deliberativo. Resolução/CD/FNDE no 38 , de 16 de julho de 2009. Dispõe sobre o atendimento da alimentação escolar aos alunos da educação básica no Programa Nacional de Alimentação Escolar - PNAE. Diário Oficial da União; Poder Executivo, 2009a.

BRASIL. Presidência da República. Casa Civil. Subchefia para Assuntos Jurídicos. Lei $\mathrm{n}^{\mathbf{0}}$ 11.947, de 17 de junho de 2009. Dispõe sobre o atendimento da alimentação escolar e do Programa Dinheiro Direto na Escola aos alunos da educação básica; altera as Leis nos 10.880, de 9 de junho de 2004, 11.273, de 6 de fevereiro de 2006, 11.507, de 20 de julho de 2007; revoga dispositivos da Medida Provisória no 2.178-36, de 24 de agosto de 2001, e a Lei no 8.913, de 12 de julho de 1994; e dá outras providências. Diário Oficial da União; Poder Executivo, 2009b.

BRASIL. Presidência da República. Casa Civil. Subchefia para Assuntos Jurídicos. Decreto ${ }^{\circ}$ 7.775, de 4 de julho de 2012. Regulamenta o art. 19 da Lei no 10.696, de 2 de julho de 2003, que institui o Programa de Aquisição de Alimentos, e o Capítulo III da Lei no 12.512, de 14 de outubro de 2011, e dá outras providências. Diário Oficial da União; Poder Executivo, 2012.

BRASIL. Ministério da Educação. Fundo Nacional de Desenvolvimento da Educação. Conselho Deliberativo. Resolução/CD/FNDE n ${ }^{\circ}$ 26, de 17 de junho de 2013. Dispõe sobre o atendimento da alimentação escolar aos alunos da educação básica no âmbito do Programa Nacional de Alimentação Escolar - PNAE. Diário Oficial da União; Poder Executivo, 2013.

BRIAND, H. The impact of AOC policy on rural development. En: SYLVANDER, B.; BARJOLLE, D.; ARFINI, F. (ed.). The socioeconomics of origin labelled products en agrifood supply chains: spatial, institutional and co-ordination aspects. $67^{\text {th }}$ EAAE seminar, le Mans, INRA, Actas y Comunicaciones, 17, Francia, 2000. p. 261-270.

BUAINAIN, A. M. A nova cara da pobreza rural: desafios para as políticas públicas. In: MIRANDA, C.; TIBÚRCIO, B. (Org.). Série Desenvolvimento Rural Sustentável, v.16, Brasília: IICA, 2012. 540p.

CONOVER, W. J. Practical Non Parametric Statistics. 3rd edition. John Wiley \& Sons, USA. 1999.

COSTA, B. A. L.; GOMES AMORIM JUNIOR, P. C.; SILVA, M. G. da. As Cooperativas de Agricultura Familiar e o Mercado de Compras Governamentais em Minas Gerais. Revista de Economia e Sociologia Rural, v. 53, p.109-126, 2015.http://dx.doi.org/10.1590/1234-567818069479005301006. 
DIMITRI, C.; OBERHOLTZER, L. Marketing U.S. Organic Foods: Recent Trends From Farms to Consumers. Economic Information Bulletin. U.S. Dept. of Agriculture: Economic Research Service. 2009.

GRISA, C. Diferentes olhares na análise de políticas públicas: considerações sobre o papel do Estado, das instituições, das ideias e dos atores sociais. Sociedade e desenvolvimento rural, v. 4, p. 96-116, 2010.

GRISA, C.; SCHMITT, C. J.; MATTEI, L. F.; MALUF, R. S.; LEITE, S. P. Contribuições do Programa de Aquisição de Alimentos à segurança alimentar e nutricional e à criação de mercados para a agricultura familiar. Agriculturas, v. 8, n. 3, p. 34-41, 2011a.

GRISA, C. O Programa de Aquisição de Alimentos (PAA) em perspectiva: apontamentos e questões para o debate. Retratos de Assentamentos, v. 13, p.137-170, 2011 b.

KROTH, D. C.; ZONIN, V. J.; COLETTI, T.; SIMÕES, W.; VON DENTZ, E. Análise da operacionalização da política de aquisição de alimentos da agricultura familiar por parte dos municípios: o caso do PNAE em três microrregiões do Oeste Catarinense. Redes (Santa Cruz do Sul. Online), v. 24, n. 1, p. 138-162, 2019. 10.17058/redes.v24i1.9697

MALUF, R. S. Mercados agroalimentares e a agricultura familiar no Brasil: agregação de valor, cadeias integradas e circuitos regionais. Ensaios FEE, Porto Alegre, v. 25, n. 1, p. 299-322, 2004.

MALUF, R. S.; MATTEI, L. F; ZIMMERMANN, S.; WESZ JUNIOR, V. In: MIRANDA, C.; B. TIBURCIO. Pobreza Rural: concepções, determinantes e proposições para a construção de uma agenda de políticas públicas. Brasília: IICA, 2011. 172p.

NUNES, E. M.; MORAIS, A. D. de; AQUINO, J. R. de; GURGEL, L. A. O Programa Nacional de Alimentação Escolar (PNAE) como política de inclusão na agricultura familiar do Nordeste do Brasil. Revista Grifos, n. 45, 2018.

RIBEIRO, A. L. de P.; CERATTI, S.; BROCH, D. T. Programa Nacional de Alimentação Escolar (PNAE) e a participação da agricultura familiar em municípios do Rio Grande do Sul. Revista Gestão e Desenvolvimento em Contexto - Gedecon, v. 1, n. 1, 2013.
SAGE, J.; GOLDBERGER, J. R. Decisions to direct market: geographic influences on conventions in organic production. Applied Geography, v. 34, p.57-65, 2012.

SAMBUICHI, R. H. R.; GALINDO, E. P.; OLIVEIRA, M. A. C. de; MOURA, A. M. M. de. Compras Públicas Sustentáveis e Agricultura Familiar: A Experiência do Programa de Aquisição de Alimentos (PAA) e do Programa Nacional de Alimentação Escolar (PNAE). In: SAMBUICHI, R. H. R. et al. (Org.). Políticas agroambientais e sustentabilidade: desafios, oportunidades e lições aprendidas. Brasília: Ipea, 2014. 273p.

SANTOS, F. dos; FERNANDES, P. F.; ROCKETT, F. C.; OLIVEIRA, A. B. A. de. Avaliação da inserção de alimentos orgânicos provenientes da agricultura familiar na alimentação escolar, em municípios dos territórios rurais do Rio Grande do Sul, Brasil. Ciência e Saúde Coletiva, v. 19, p. 1429-1436, 2014. http://dx.doi.org/10.1590/1413-

81232014195.14982013 .

SAQUET, M. A.; SOUZA, P. de; SANTOS, R. A. dos. Agricultura familiar agroecológica em Itapejara d'Oeste-PR. Revista da ANPEGE, v. 6, p. 53-57, 2010.

SCHRÖETTER, M.R.; BÜTTENBENDER, P. L.; GRAEF, C. E. Cooperativas da Agricultura Familiar e a Merenda Escolar: Produção, Comercialização e Consumo na Região Fronteira Noroeste. 2013.

SOUZA-ESQUERDO, V. F. de; BERGAMASCO, S. M. P. P. Análise sobre o acesso aos programas de políticas públicas da agricultura familiar nos municípios do circuito das frutas (SP). Revista de Economia e Sociologia Rural, v. 52, p. 205222, 2014. 20032014000600011

TRICHES, R. M.; SCHNEIDER, S. Alimentação escolar e agricultura familiar: reconectando consumo a produção. Saúde e Sociedade, v. 19, p. 933-939, 2010.

TRICHES, R. M.; SIMONETTI, M. G.; CASSARINO, J. P.; BACCARIN, J. G.; TEO, C. R. P. A. Condicionantes e limitantes na aquisição de produtos da agricultura familiar pelo Programa de Alimentação Escolar no estado do Paraná. Redes (Santa Cruz do Sul. Online), v. 24, n. 1, p. 118-137, 2019. 10.17058/redes.v24i1.11713.

ZOLDAN, P. C.; MIOR, L. C. Produção orgânica na agricultura familiar de Santa Catarina. Florianópolis: Epagri, 2012. 94p. 